Case report

\title{
Celiac disease with subnephrotic range proteinuria: an enigma unresolved
}

\author{
Nitya Nand, Deepak Jain*, Raghunandan SK, Kajaree Giri, Promil Jain \\ Department of Medicine, Pt. B.D. Sharma University of Health Sciences, Haryana, India
}

\begin{abstract}
Celiac disease, an autoimmune mediated enteropathy is associated with a variety of autoimmune diseases involving various organ systems to various extents, among which the renal involvement, although rare, needs a special reference. According to the literature, celiac disease is mostly associated with $\lg A$ nephropathy. Lupus nephritis and focal segmental glomerulosclerosis type of renal involvement has also been described to occur in association with celiac disease but many case reports have described patients of celiac disease presented with nephrotic or subnephrotic range proteinuria which have remained unexplained till date. Here we present such a case of with subnephrotic range proteinuria with normal kidney biopsy whose association has been explored.
\end{abstract}

Keywords: celiac disease; proteinuria; autoimmune diseases

\section{Introduction}

Celiac disease is an immune mediated enteropathy. It is often associated with some autoimmune diseases such as type 1 diabetes mellitus, autoimmune thyroid disorders, Sjögren syndrome and $\lg A$ nephropathy [1]. Proteinuria occurring in a poorly controlled celiac disease patient is associated with the diseases involving the kidneys with similar ethology and pathogenesis as that of the celiac disease such as IgA nephropathy, systemic lupus erythematosus (SLE) and, very rarely, focal segmental glomerulosclerosis type of renal involvement [2-4]. Also there are rare case report of proteinuria occurring in $C D$ patients whose diagnoses has remained enigmatic till date. Here we report a rare case of celiac disease presenting with history of persistent proteinuria in the subnephrotic

Received: May 2018; Accepted after review: September 2018; Published: December 2018.

${ }^{*}$ Corresponding author: Deepak Jain, Department of Medicine, Pt. B.D. Sharma University of Health Sciences, ROHTAK-124001 (Haryana) India.

Email: jaindeepakdr@gmail.com range without any histological change in the kidneys and patient responded to a strict gluten free diet.

\section{Case report}

A 12 year-old female student, known case of celiac disease for 5 years on partial gluten free diet for last few months presented with complaints of diarrhea on and off for 2 months, pain abdomen for 1 month and swelling bilateral feet and face for 2 weeks. She did not have any history of fever, sore throat, joint pains, rash over the body, vomiting, burning micturition, hematuria, photosensitivity, apthous ulcers or alopecia. She denied history of any other chronic illness in the past. Family and personal history were not contributory.

On examination, she was normally built, moderately nourished, alert, conscious, cooperative and oriented to time, place and person. Her blood pressure was 110/82 $\mathrm{mmHg}$, Pulse rate $86 / \mathrm{min}$, regular and respiratory rate was $18 / \mathrm{min}$. Patient was afebrile, had mild pallor and pedal edema and 
there was no icterus, cyanosis, clubbing, lymphadenopathy. Patient's height was 152.4 $\mathrm{cm}$ (between the $25^{\text {th }}$ and $50^{\text {th }}$ percentile of normal reference for the age of the patient), weight was $35 \mathrm{~kg}$ (between the $10^{\text {th }}$ and $25^{\text {th }}$ percentile of normal reference for the age of the patient) and a BMI of $15.15 \mathrm{~kg} / \mathrm{m} 2$. Patient had as yet not attained menarche and had a sexual maturity of Tanner's stage 2 . Rest of the systemic examination was within normal limits.

On routine investigations, complete hemogram showed mild anemia with hemoglobin of $8.9 \mathrm{~g} / \mathrm{dl}$. Blood sugar, lipid profile, kidney and thyroid function tests were within normal limits. Serum proteins were 5.9 $\mathrm{g} / \mathrm{dl}$, serum albumin was $3.1 \mathrm{~g} / \mathrm{dl}$, serum calcium was $9.6 \mathrm{mg} / \mathrm{dl}$, serum phosphorus 4.5 $\mathrm{mg} / \mathrm{dl}$, serum alkaline phosphatase was raised (182 IU/L) and serum Vit $D$ was low (17 $\mathrm{ng} / \mathrm{ml})$. Urine complete examination by dipstick method showed proteinuria on three different occasions. The proteinuria quantified over 24 hours was found to be $1.4 \mathrm{~g} /$ day and $1.5 \mathrm{~g} /$ day on two different occasions from outside reports done one month back and it was $1.6 \mathrm{~g} /$ day during current admission. Urine examination for dysmorphic red blood cells and casts done on two different occasions were negative. Urine culture reports were sterile repeatedly. Serum $\lg$ A tissue transglutaminase antibodies were positive with an absolute value of 54 $\mathrm{u} / \mathrm{ml}$. ASO titre was $<120 \mathrm{u} / \mathrm{ml}$. Chest X-Ray, ECG and echocardiography findings were within normal limits. Ultrasonography of the abdomen revealed normal sized kidneys with maintained corticomedullary differentiation. Patient was non-reactive for HIV, hepatitis B and hepatitis $C$ antibodies. An intestinal biopsy revealed Celiac disease Marsh grade 3 suggesting of poorly controlled disease status (Figure 1).

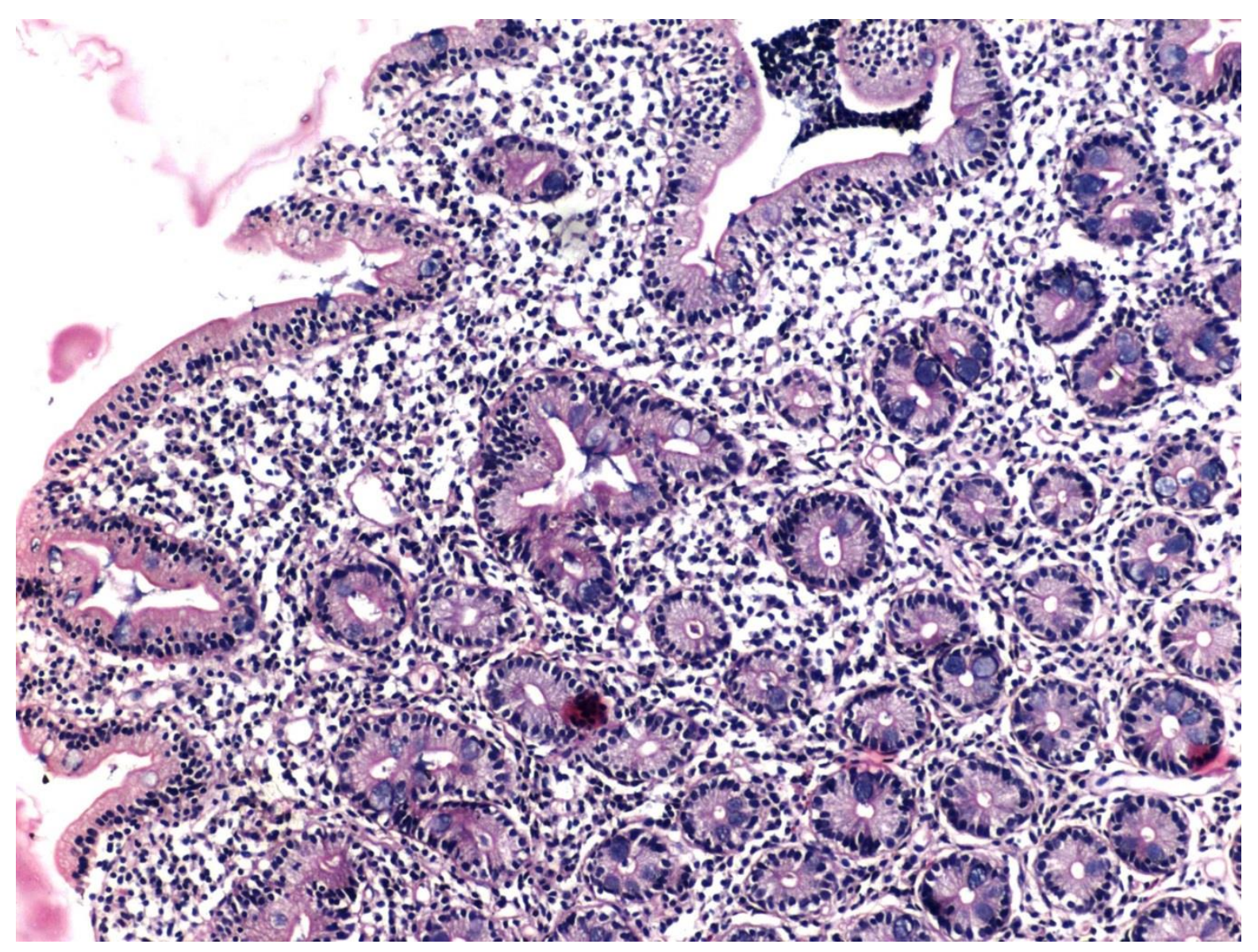

Fig. 1. Duodenal biopsy showing Marsh grade 3 changes of the mucosa (HE, x200).

ANA was positive with a titre of 1:80 while Anti dsDNA and anti-Sma were negative and serum C3, C4 and CRP levels were within normal limits. Renal biopsy showed normal 
histology on the light microscopy and did not show any immune deposits on immunofluorescence study (Figure 2). Patient was started on a strict gluten free diet and angiotensin converting enzyme inhibitors with which she showed a drastic improvement. On follow-up after 3 months, she was doing well and her proteinuria was about $500 \mathrm{mg} /$ day.

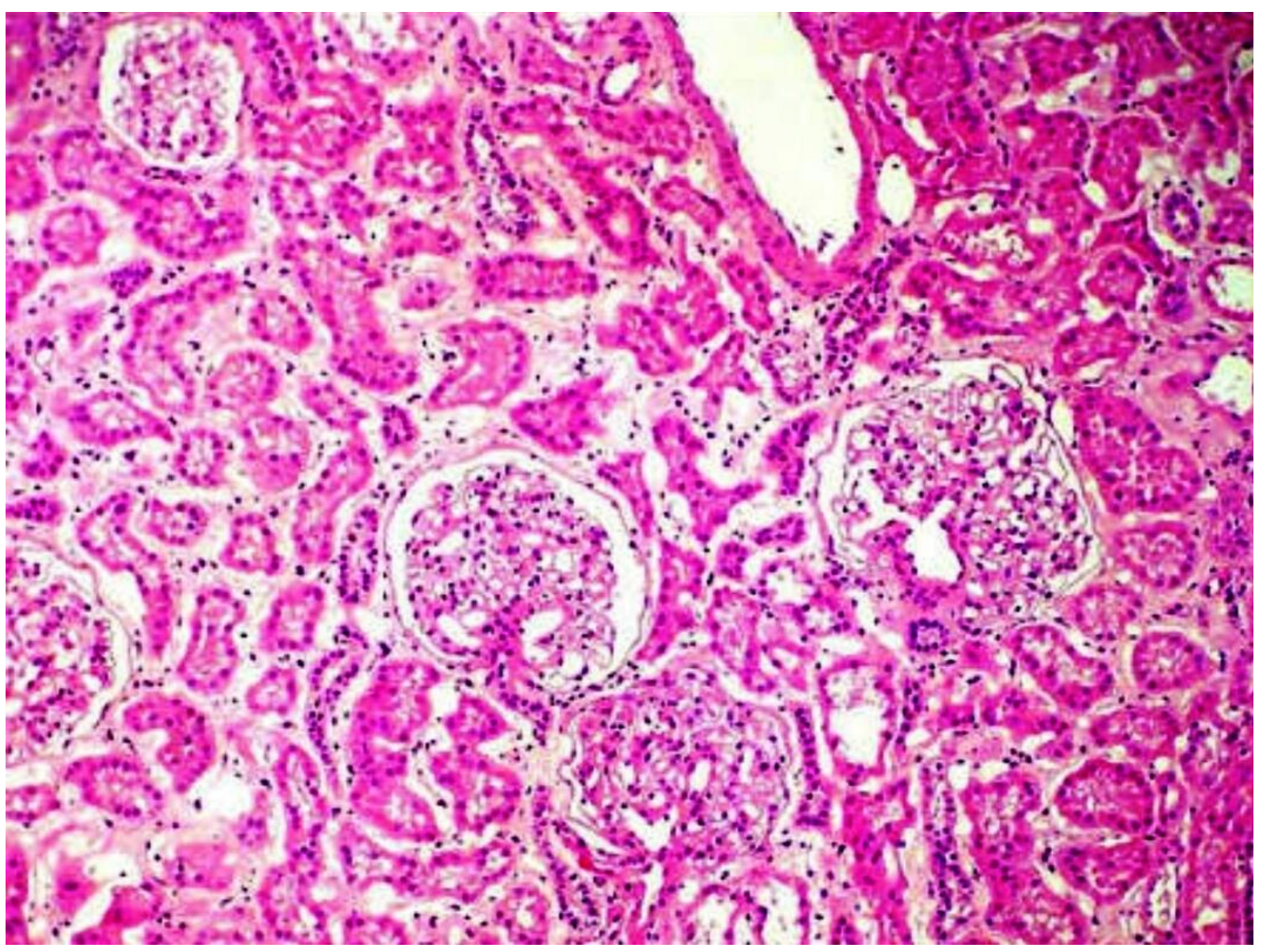

Fig. 2. Renal biopsy showing normal histology (HE, x200).

\section{Discussions}

Celiac disease is one of the most common diet mediated inflammatory enteropathy of an autoimmune etiology that occurs in genetically predisposed people of all ages. It is associated with an increased risk of developing other autoimmune conditions such as type 1 diabetes mellitus, thyroid disease, Sjögren's syndrome and $\lg A$ nephropathy. Kidney involvement in association with celiac disease has been seen in the order of decreasing frequencies in $\lg A$ nephropathy, SLE, focal segmental glomerulosclerosis in the literature [2-4].

$\lg$ A nephropathy which usually presents as a primary glomerular disorder characterized by episodic hematuria shows deposition of $\lg \mathrm{A}$ in the mesangium is also known to occur in association with celiac disease due to the presence of high levels of $\lg A$ antibodies against the food antigen, gliadin in these patients [5]. But in our case due to the absence of hematuria and the classical features such as mesangial segmental hypercellularity, increased mesangial matrix and granular $\lg \mathrm{A}$ deposits in the mesangium, the possibility of $\lg A$ nephropathy was ruled out.

Although very rare, an association of celiac disease with systemic lupus erythematosus has been observed. The prevalence of the disease is about 1 in 1000 , or even as low as 1 in 100 with cases ranging from classic celiac to atypical or even silent celiac disease [6, 7]. Presence of anti-tissue 
transglutaminase antibodies in patients of SLE and ANA and anti dsDNA in about 10-15\% patients of celiac disease had made researchers look into the associations between the two diseases but there are only limited number of case reports of concomitant SLE with celiac disease [8]. In literature, there are case reports quoting that in patients of celiac disease with proteinuria and kidney biopsy changes mimicking SLE; biopsy changes reverse with strict gluten free diet [9]. However in our patient, absence of clinical features suggestive of SLE and anti-dsDNA with normal serum complement levels, normal histological and immunofluorescence study of the renal biopsy tissue ruled out the diagnosis of SLE. There is limited literature revealing the association of focal segmental glomerulosclerosis with celiac disease across the world till date [4].

As recently hypothesized, in patients with celiac disease, the increased intestinal permeability or "leaky gut" acts as a driving force in bacterial translocation and subsequent circulation of lipopolysaccharides which in turn cause podocyte damage in the kidneys [10, 11]. Vitamin $D$ deficiency which occurs in these patients also contributes to enhance this lipopolysaccharide induced podocyte damage, resulting in minimal change disease-like lesions. The food sensitivity in celiac disease may also cause immune dysregulation by over expressing various interleukins, cytokines and nuclear kappa B pathway and result in overactive response against podocytes resulting in proteinuria $[12,13]$.

\section{References}

1. Farrell RJ, Kelly CP. Celiac sprue. $N$ Engl $J$ Med 2002; 346:180-8.

2. Koivuviita N, Tertti R, Heiro M, Metsarinne K. A case report: a patient with $\lg A$ nephropathy and celiac disease. Complete clinical remission following gluten free diet. Nephrol Dial Transplant Plus 2009; 2:161-163.

3. Hugh J, Freeman, MD. Adult celiac disease followed by onset of systemic lupus erythematosus. J Clin Gastroenterol 2008; 42:252-255.

4. Harbi $H$, Abdelsalam MS, Abdulshakour A, Alfurayh O. Celiac disease and focal segmental
The proteinuria in our patient had decreased in response to gluten free diet and angiotensin converting enzyme inhibitors that were used empirically in our patient. We contribute the presence of proteinuria in our case to autoimmunity and the other mechanisms described above. Introduction of gluten may be the causative factor of renal involvement based on immune complex pathogenesis. However the exact pathophysiology of rare association still needs to be elucidated.

\section{Conclusion}

This case report emphasizes the fact that the grey area in this field requires extensive studies and thus the preventive strategies to be evolved for the betterment of these patients. Long term follow-up of such patients and repeated biopsies may establish that the proteinuria and celiac disease may be more closely linked than is currently appreciated.

\section{Consent}

Patient consent form has been procured prior to the case report study after fully disclosing all points in local and English language.

\section{Competing interests}

All the authors have declared no conflict of interest to the work carried out in this paper. glomerulosclerosis: Is it a fortuity finding? World J Nephrol Urol 2015; 4(4):264-266.

5. Pierucci A, Fofi C, Bartoli B, et al. Antiendomysial antibodies in Berger's disease. Am J Kidney Dis 2002; 39:1176-1182.

6. Feighery C. Fortnightly review: coeliac disease. BMJ 1999; 319(7204):236-239.

7. Maki M, Mustalahti K, Kokkonen J, et al. Prevalence of Celiac disease among children in Finland. N Engl J Med 2003; 348(25):25172524.

8. Marai I, Shoenfeld $\mathrm{Y}$, Bizzaro N, et al. Ig A and Ig $G$ tissue transglutaminase antibodies in 
systemic lupus erythematosus. Lupus 2004; 1(4)3:241-244.

9. Hadjivassiliou M, Sanders DS, Grunewald RA, Akil M. Gluten sensitivity masquerading as systemic lupus erythematosus. Ann Rheum Dis 2004; 63:1501-1503.

10. Bischoff SC, Barbara G, Buurman W, et al. Intestinal permeability: A new target for disease prevention \& therapy. BMC Gastroenterol 2014; (14):189.
11. Lau WL, Kalantar-Zadeh, K., Vaziri ND. The gut as a source of inflammation in chronic kidney disease. Nephron 2015; 130(2):92-98.

12. Shimada $M$, Ishimoto $T$, Lee $P Y$, et al. Toll-like receptor 3 ligands induce CD80 expression in human podocytes via an NF-kB-dependent pathway. Nephrol Dial Transplant 2012; 27(1):81-89.

13. Vasylyeva TL, Singh R. Gut microbiome and kidney disease in pediatrics: Does connection exist? Front Microbiol 2016; 7:235. 